\title{
Prohibitin 2 deficiency impairs cardiac fatty acid oxidation and causes heart failure
}

Dechao Wu ${ }^{1,2}$, Chongshu Jian', Qi Peng $\mathbb{D}^{1}$, Tingting Hou', Keling Wu², Bizhi Shang², Minglei Zhao², Yanru Wang ${ }^{1}$, Wen Zheng ${ }^{1}$, Qi Ma', Chuan-Yun Li', Heping Cheng $\mathbb{1}^{1}$, Xianhua Wang ${ }^{1}$ and Ling Zhao ${ }^{2}$

\begin{abstract}
Fatty acids are the most major substrate source for adult cardiac energy generation. Prohibitin 2 (PHB2), a highly conserved protein located in mitochondrial inner membrane, plays key roles in cellular energy metabolic homeostasis. However, its functions in regulating cardiac fatty acid metabolism have remained largely unknown. Our study demonstrates that cardiac-specific knockout of Phb2 leads to accumulation of lipid droplets and causes heart failure. Mechanistically, ablation of PHB2 impairs cardiac fatty acid oxidation (FAO) through downregulating carnitine palmitoyltransferase1b (CPT1b), a rate-limiting enzyme of cardiac mitochondrial FAO. Moreover, overexpression of CPT1b alleviates impaired FAO in PHB2-deficient cardiomyocytes. Thus, our study provides direct evidence for the link between PHB2 and cardiac fatty acid metabolism. Our study points out that PHB2 is a potential FAO regulator in cardiac mitochondrial inner membrane, as well as the connection between PHB2 and CPT1b and their relationships to cardiac pathology especially to cardiac fatty acid metabolic disorder.
\end{abstract}

\section{Introduction}

Despite decades of research, heart failure is still a leading cause of morbidity and mortality in modern society ${ }^{1}$. It is a complex clinical syndrome, in which abnormality in cardiac pump function leads to inadequate oxygen supply and perturbed energy metabolism for maintaining normal requirement of cardiac contractility ${ }^{2}$. A variety of mechanisms have been involved in the pathogenesis of heart failure, including metabolic disorder, mitochondrial dysfunction, autophagy, apoptosis, and genetic or epigenetic dysregulation ${ }^{3-5}$. Better understanding the underlying mechanisms of heart failure will provide potential therapeutic targets.

A high rate of ATP production and turnover is required for maintaining normal cardiac functions ${ }^{2}$. As the main

\footnotetext{
Correspondence: Xianhua Wang (xianhua@pku.edu.cn) or

Ling Zhao (zhaoling6@mail.sysu.edu.cn)

${ }^{1}$ State Key Laboratory of Membrane Biology, Beijing Key Laboratory of

Cardiometabolic Molecular Medicine, Peking-Tsinghua Center for Life Sciences, Institute of Molecular Medicine, Peking University, Beijing, China

${ }^{2}$ State Key Laboratory of Ophthalmology, Zhongshan Ophthalmic Center, Sun

Yat-sen University, Guangzhou, China
}

Edited by $\mathrm{G}$. Melino source of energy, mitochondrial abnormalities in ATP production may contribute to the pathology of heart failure $^{6,7}$. Prohibitin 2 (PHB2), a highly conserved protein located in mitochondrial inner membrane, plays key roles in cellular energy metabolic homeostasis ${ }^{8,9}$. PHB2 is critical in structural and functional integrity of mitochondria, stability of mitochondrial genome, modulation of mitochondrial dynamics, mitochondrial cristae morphology, respiratory supercomplex formation, mitophagy and mitochondrial quality control ${ }^{10-15}$. However, the function of cardiac PHB2 is less well known, especially in fatty acid metabolic regulation.

Fatty acids are the most major substrate source for adult cardiac ATP generation ${ }^{16,17}$. Fatty acids are mainly fueled via $\beta$-oxidation to produce ATP in mitochondria ${ }^{18}$. Severe perturbation in cardiac fatty acid metabolism homeostasis and energy production have been regarded as a consistent feature of heart failure ${ }^{19,20}$. For instance, carnitine palmitoyltransferase-1b (CPT1b), which is the predominant isoform expressed in adult heart, is one of the most important components in controlling fatty acid $\beta$-oxidation $(\mathrm{FAO})^{21,22}$. CPT1b deficiency results in 
impaired cardiac fatty acid oxidation under pressureoverload and exacerbation of cardiac pathology $y^{23}$. As mechanisms underlying the regulation of cardiac FAO are complex, signaling pathways of modulating cardiac FAO are still incompletely elucidated.

Loss of Phb2 in Caenorhabditis elegans and mice leads to embryonic lethality ${ }^{24,25}$. Several tissue specific $P h b 2$ knockout mice have been used to explore its functions in physiological and pathological processes ${ }^{26-29}$. In forebrainspecific PHB2-deficient mice, tau hyper-phosphorylation and neurodegeneration were observed ${ }^{29}$. $\beta$-cell-specific knockdown of PHB2 resulted in impaired $\beta$-cell function and diabetes ${ }^{28}$. Deletion of PHB2 in podocytes led to the development of glomerulosclerosis due to PHB2's extramitochondria role in the assembly of the slit diaphragm protein-lipid supercomplex ${ }^{27}$. Hepatocyte-specific Phb2 knockout mice exhibited liver failure and impaired gluconeogenesis ${ }^{26}$. The above studies demonstrate that PHB2 is essential for maintaining normal organ function. Thus, the generation of a cardiac specific Phb2 knockout mice should greatly help to explore its functions in cardiac energy metabolism in vivo as well.

In our study, we generated cardiac specific Phb2 knockout mice that exhibited fatty acid oxidation disturbance, mitochondrial dysfunction, and eventually heart failure and lethality, demonstrating that PHB2 is essential for maintaining normal cardiac metabolic functions. Our study also explored whether PHB2 acts as a mitochondrial inner membrane FAO regulator and the relationships between PHB2 and CPT1b in cardiac fatty acid metabolism.

\section{Results}

\section{Cardiac-specific deletion of $\mathrm{Phb2}$ leads to dilated cardiomyopathy and heart failure}

As whole-body knockout of Phb2 was embryonic lethal ${ }^{24,25}$, we generated a cardiac-specific Phb2 knockout (Phb2 cKO) mouse model wherein exon 4 of Phb2 was flanked by LoxP sites. The Phb $2^{\text {flox/flox }}$ mice were crossbred with Mlc2v-Cre mice to allow cardiac-specific deletion of Phb2 (Fig. S1). Compared with wild type (WT) littermates, protein expression of cardiac PHB2 was decreased by $80 \%$ in $P h b 2$ cKO mice at 6 weeks of age (Fig. 1a). Cardiac Phb2 cKO mice exhibited typical phenotypes of dilated cardiomyopathy and heart failure. Compared with WT group, the lifespan of Phb2 cKO mice was markedly shortened, with sudden death beginning at around 10 weeks of age and a maximal lifespan at 12 weeks of age (Fig. 1b). Ventricular dilation and thinner posterior wall thickness were observed in hematoxylin and eosin (HE) staining of cardiac vertical sections of Phb2 cKO mouse hearts at 8 weeks of age (Fig. 1c). Masson staining showed that cardiac ablation of PHB2 induced extensive cardiac fibrosis, and the fibrotic area increased by approximately two-fold compared with WT at 8 weeks of age (Fig. 1d). Moreover, cardiac echocardiography (ECHO) measurements demonstrated cardiac systolic dysfunctions in $\mathrm{Phb} 2 \mathrm{cKO}$ mice at 8 weeks of age (Fig. 1e), showing both ejection fraction (EF) and fractional shortening (FS) diminished by $70 \%$ compared with WT (Fig. 1f). ECHO analysis indicated Phb2 cKO mouse hearts exhibited enlarged left ventricular inner diameter, increased left ventricular volume and reduced posterior wall thickness during systolic state compared with WT group (Fig. 1g-i). Meanwhile, compared with WT, the heart weight/body weight ratio increased by $34.3 \%$, and the heart weight/tibia length ratio increased by $19.7 \%$ in Phb2 cKO mice at 8 weeks of age (Fig. 1j). In addition, it was found that Phb2 cKO mice exhibited normal cardiac systolic functions at 4 and 6 weeks of age (Fig. S2A, B, Table S1) and there was no difference in body weight between WT and $P h b 2 \mathrm{cKO}$ mice at 8 weeks of age (Fig. S2C). Thus, cardiac specific Phb2 knockout mouse model is generated and cardiac ablation of Phb2 results in heart failure shown by shortened lifespan, dilated left ventricle, interstitial fibrosis, and systolic dysfunctions.

\section{Impaired cardiac energy metabolism and mitochondrial dysfunction are caused by PHB2 ablation}

Cardiac energy metabolism was investigated in 6-weekold Phb2 cKO mice. Cardiac ATP levels measured with luciferin assay in 6-week-old WT and Phb2 cKO mouse hearts revealed that PHB2 ablation resulted in lower cellular ATP content (Fig. 2a). The mitochondrial maximal respiration measured by Seahorse assay was prominently suppressed in isolated cardiomyocytes from $\mathrm{Phb2} \mathrm{cKO}$ mice (Fig. 2b). As mitochondrial morphological changes are also related with cardiac dysfunctions ${ }^{30}$, transmission electron microscopy was used to examine cardiac mitochondrial ultrastructure. Compared with WT mice, swollen cardiac mitochondria with few lamellar cristae were observed in Phb2 cKO mouse hearts (Fig. 2c). Strikingly, lipid droplets (LD) were widely spread around cardiac mitochondria in Phb2 cKO mouse hearts (Fig. 2c, pyknotic and black circles represented LD), which pointed out that PHB2 might directly regulate cardiac fatty acid metabolism.

We also checked whether PHB2 ablation result in cardiac mitochondrial dysfunction in 6-week-old Phb2 cKO mice. Using the potential-sensitive fluorescent probe tetramethyl rhodamine methyl ester (TMRM), a significantly decreased mitochondrial membrane potential $(\Delta \Psi \mathrm{m})$ was found in isolated cardiomyocytes from $\mathrm{Phb} 2 \mathrm{cKO}$ mice (Fig. 2d). Fluorescent probes mitoSOX and DCF were applied to detect mitochondrial and cytosolic ROS, respectively. Both mitochondrial and cytosolic ROS production were markedly elevated in isolated cardiomyocytes from Phb2 cKO mice compared with WT (Fig. 2e, f). 


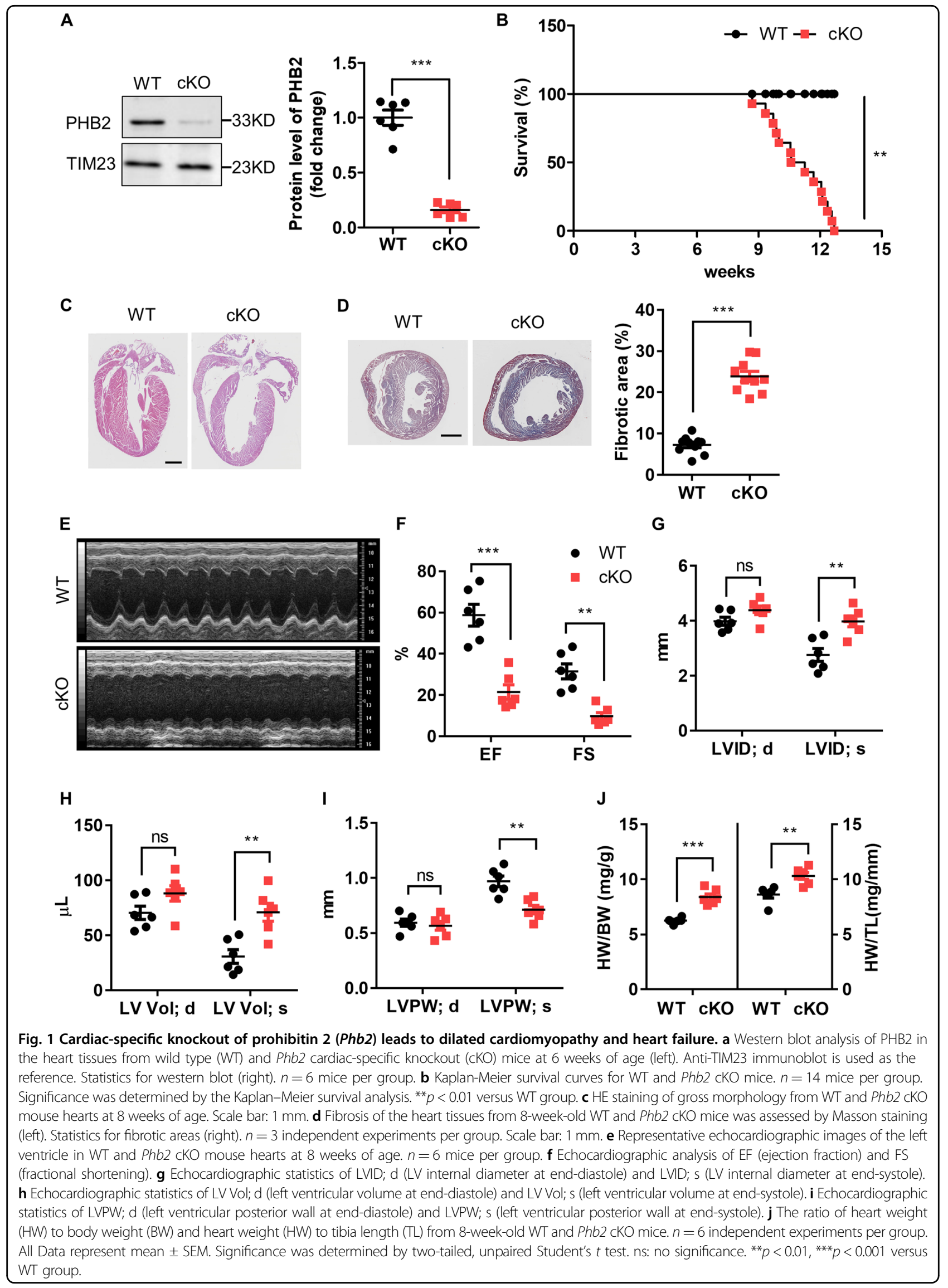



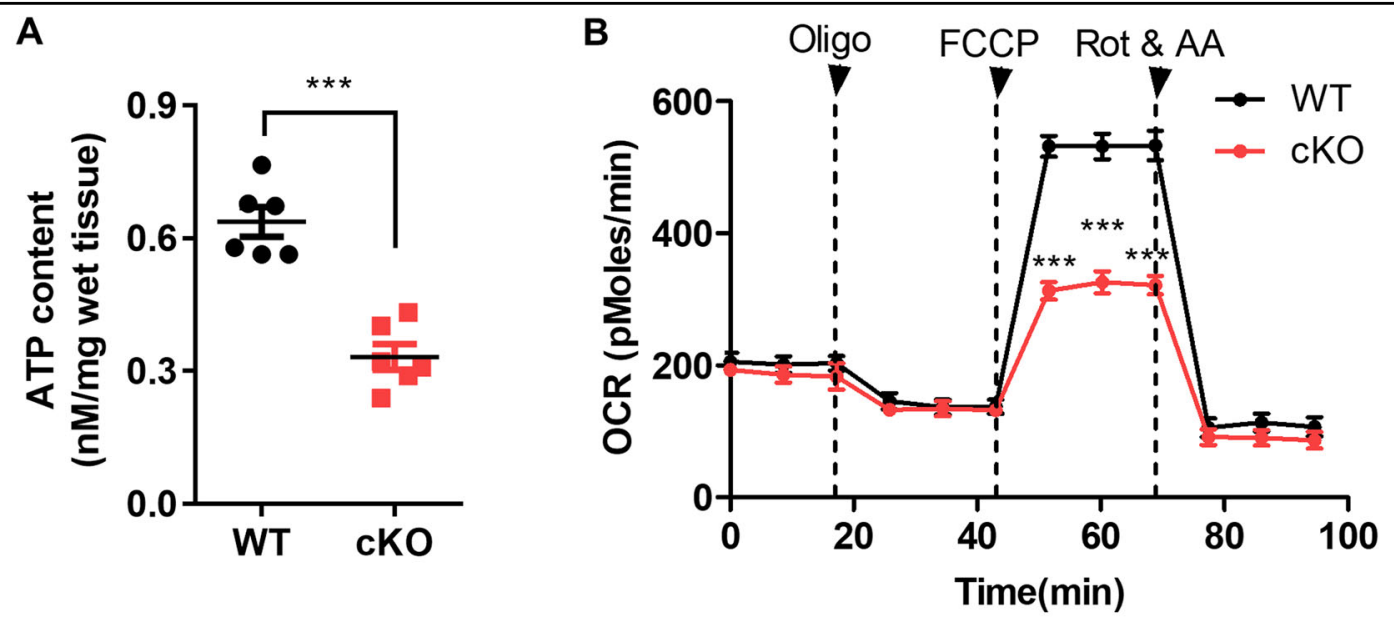

C

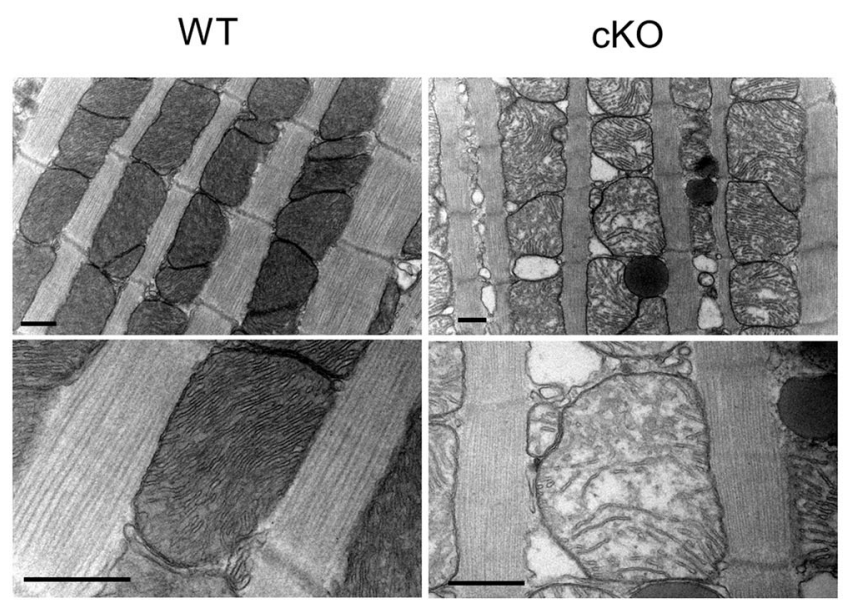

D

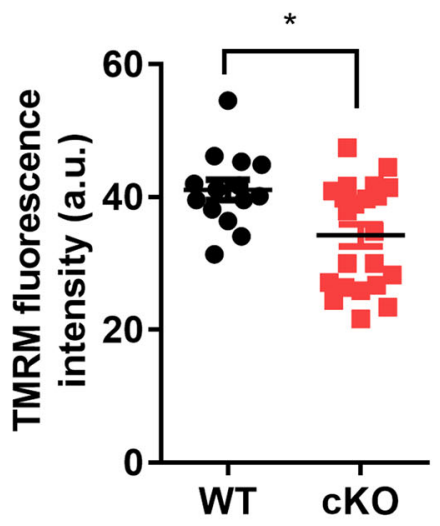

E

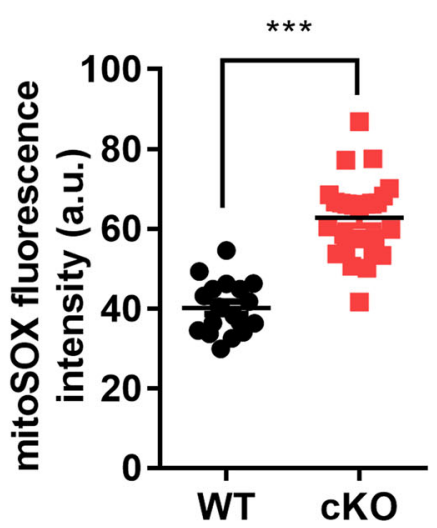

$\mathbf{F}$

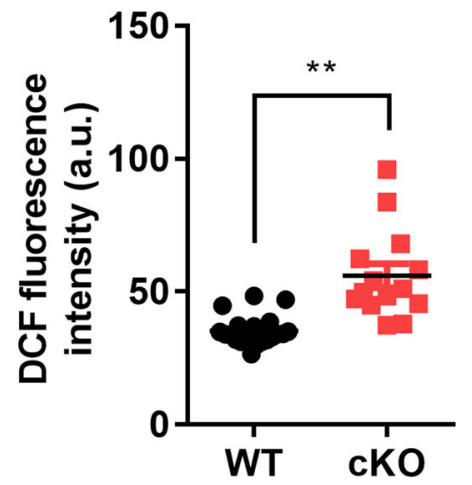

Fig. 2 Impaired cardiac energy metabolism and mitochondrial dysfunction are caused by PHB2 ablation. a Cardiac ATP levels measured with luciferin assay in 6-week-old WT and Phb2 cKO mouse hearts. $n=6$ mice per group. $\mathbf{b}$ Measurement of oxygen consumption rate (OCR) in isolated cardiomyocytes from 6-week-old WT and Phb2 CKO mice. Arrowheads indicate the time points of adding Oligo (oligomycin, 1 mm), FCCP (carbonyl cyanide 4-(trifluoromethoxy) phenylhydrazone, $1 \mu \mathrm{m}$ ), Rot (rotenone, $1 \mu \mathrm{m}$ ) and AA (antimycin, $1 \mu \mathrm{m}$ ). $n=3$ mice per group. $\mathbf{c}$ Electron micrographs of mitochondrial morphology in left ventricular tissues from 6-week-old WT and Phb2 cKO mice. Scale bar: $0.5 \mu \mathrm{m}$. $\mathbf{d}$ Statistics of mitochondrial membrane potential measured with TMRM staining in isolated cardiomyocytes from 6-week-old WT and Phb2 cKO mice. $n=80-100$ cells from six mice per group. e Statistics of mitochondrial ROS levels measured with mitoSOX staining in isolated cardiomyocytes from 6-week-old WT and Phb2 cKO mice. $n=80-100$ cells from six mice per group. $\mathbf{f}$ Statistics of cytosolic ROS levels measured with DCF staining in isolated cardiomyocytes from 6week-old WT and Phb2 cKO mice. $n=80-100$ cells from six mice per group. All Data represent mean \pm SEM. Significance was determined by twotailed, unpaired Student's $t$ test. ${ }^{*} p<0.05,{ }^{* *} p<0.01,{ }^{* * *} p<0.001$ versus WT group. 

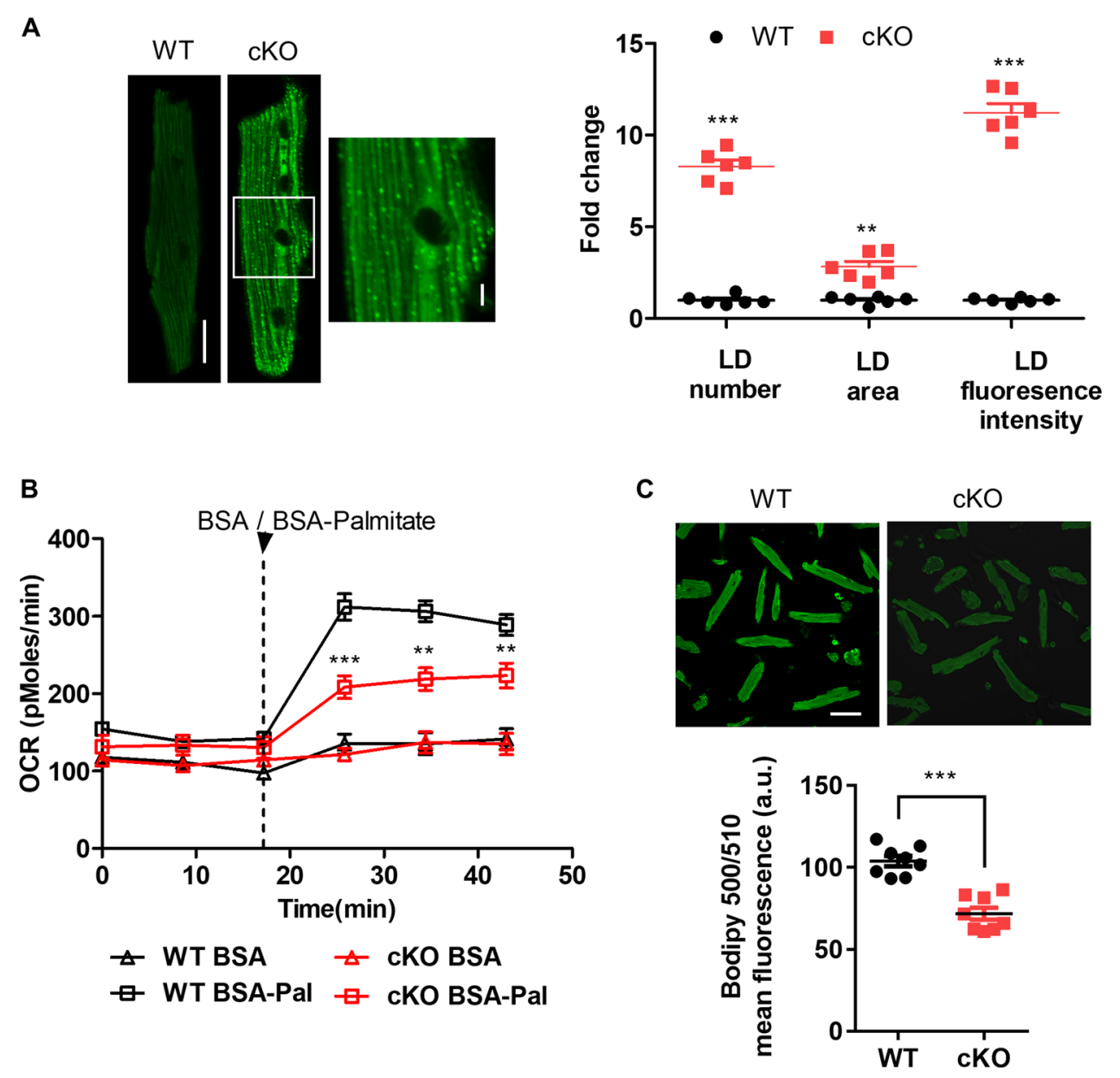

Fig. 3 Impaired fatty acid oxidation (FAO) and cardiac lipid accumulation are caused by PHB2 ablation. a Representative confocal images of lipid droplets (LD) visualized by BODIPYTM 493/503 in isolated cardiomyocytes from 6-week-old WT and Phb2 cKO mice (left). Scale bar: $20 \mu \mathrm{m}$. The enlargement is the magnification of the boxed inset shown in left panel. Scale bar: $5 \mu \mathrm{m}$. Statistics of fold change of LD number, area and fluorescence intensity per cardiomyocyte (right). $n=80-100$ cells from six mice per group. b Measurement of fatty acid oxidation (FAO) in isolated cardiomyocytes from 6-week-old WT and Phb2 cKO mice. Arrowhead indicates the time points of adding BSA (0.17 mM) or BSA-Pal (Palmitate) $(1 \mathrm{mM}) . n=3$ mice per group. c Representative confocal images of fatty acid uptake (FAU) visualized by BODIPYTM 500/510 in isolated cardiomyocytes from 6-week-old WT and Phb2 CKO mice (top). Statistics of FAU levels (bottom). $n=80-100$ cells from six mice per group. Scale bar: $50 \mu m$. All Data represent mean \pm SEM. Significance was determined by two-tailed, unpaired Student's $t$ test. ns: no significance. ${ }^{* *} p<0.01,{ }^{* * *} p<0.001$ versus WT group.

Herein, cardiac mitochondrial dysfunction caused by PHB2 deficiency might partially account for heart failure in Phb2 cKO mice. Collectively, these results indicated that impaired cardiac energy metabolism and mitochondrial dysfunction are caused by PHB2 ablation.

\section{Impaired fatty acid oxidation and cardiac lipid accumulation are caused by PHB2 ablation}

Unbalanced uptake and oxidation of fatty acids will lead to lipid accumulation in heart tissues, which is also a crucial reason for the occurrence and progression of heart failure ${ }^{31}$. BODIPY 493/503 staining was used for cardiac lipid measurements ${ }^{32,33}$. In our study, LD accumulation was significantly increased, shown by higher LD number, area and higher fluorescence intensity in isolated cardiomyocytes from Phb2 cKO mice at 6 weeks of age (Fig. 3a). Additionally, Oil Red O staining was performed to examine lipid accumulation in heart tissues and results indicated that much more neutral lipid accumulated in 6-week-old Phb2 cKO mice compared with WT group (Fig. S3).

We further investigated the underlying correlations between PHB2 and cardiac LD accumulation by testing fatty acid oxidation (FAO) and fatty acid uptake (FAU). It was interesting that both FAO and FAU were markedly reduced in isolated cardiomyocytes from $\mathrm{Phb} 2 \mathrm{cKO}$ mice. Cardiac FAO was strongly inhibited by PHB2 ablation, which was shown by a much lower FAO of Phb2 cKO than that of WT with BSA-Palmitate treatment (Fig. 3b). 
BODIPY 500/510 staining was applied to examine FAU in cardiomyocytes $^{34}$. Results indicated that cardiac FAU was also significantly decreased in $\mathrm{Phb} 2 \mathrm{cKO}$ mice compared to WT (Fig. 3c). Thus, we suppose that impaired FAO by PHB2 ablation might be a more dominant cause for cardiac lipid accumulation, though decreased FAU might alleviate some cardiac lipid metabolic disorder.

\section{Proteomic profiles reveal that CPT1b is downstream of PHB2 signaling pathway in cardiac FAO}

To explore the key components downstream of PHB2 deficiency in cardiac fatty acid metabolic homeostasis, we performed quantitative proteomic analysis of WT and Phb2 cKO mouse hearts at 6 weeks of age. Among 4172 proteins, 931 differentially expressed proteins (fold change $\geq 1.2$, $P$ value $<0.05)$ were identified to be associated with distinct biological processes. 418 proteins were significantly upregulated and 513 proteins were significantly downregulated in Phb2 cKO mouse hearts. KEGG pathways analysis revealed that PHB2 deficiency resulted in a prominent disturbance of oxidative phosphorylation and metabolic pathways (Fig. 4a). Several proteins involved in heart failure were downregulated, which were found with the terms "Dilated cardiomyopathy" and "Cardiac muscle contraction", such as TPM1 (Tropomyosin 1), TNNT2 (Troponin T2), ACTC1 (Actin, alpha, cardiac muscle 1) (Fig. 4b). As expected, the most enriched pathways were related to mitochondrial function, including ATP biosynthesis, electron transport chain and oxidative phosphorylation (Fig. 4c). Proteomics profiles also revealed that metabolic pathways, especially fatty acid metabolism, was altered by PHB2 deficiency. FABP3 (Fatty acid-binding protein 3), FABP4 (Fatty acid-binding protein 4), CD36 (Cluster of differentiation 36), CPT1b (Carnitine palmitoyltransferase 1b), CPT2 (Carnitine palmitoyltransferase 2), ACADM (Medium-chain specific acyl-CoA dehydrogenase), ACADVL (Very long-chain specific acyl-CoA dehydrogenase), ACACB (Acetyl-CoA carboxylase 2), and ACAA2 (3-ketoacyl-CoA thiolase) were all found to be downregulated in $\mathrm{Phb} 2 \mathrm{cKO}$ mouse hearts (Fig. 4d). Decreased expression of FABP3, CD36 and CPT1b in $\mathrm{Phb2}$ cKO mouse hearts was validated by western blot analysis (Fig. 4e). As CPT1b is a rate-limiting enzyme for mitochondrial fatty acid $\beta$-oxidation in adult mouse cardiomyocyte $^{23}$, our results indicate CPT1b might be a downstream component of the PHB2 signaling pathway in regulating cardiac FAO.

\section{Impaired cardiac FAO is caused by PHB2 knockdown in NRVMs}

We used isolated neonatal rat ventricular myocytes (NRVMs) to explore the underlying mechanisms of PHB2's regulation of cardiac fatty acid metabolism. In isolated NRVMs, PHB2 knockdown via siRNA (si-PHB2) could induce $\mathrm{LD}$ accumulation compared with the negative control (NC) group, reflected by higher LD number, area and higher fluorescence intensity per NRVM (Fig. 5a). FAO of BSA-Palmitate group in si-PHB2 NRVMs was markedly lower than that in NC NRVMs (Fig. 5b), which revealed that PHB2 deficiency significantly inhibited FAO in NRVMs. Decreased expression of CPT1b was detected in si-PHB2 NRVMs (Fig. 5c). As CPT1a is the predominant isoform in fetal cardiomyocytes ${ }^{21,35}$, we also checked its expression in si-PHB2 treated NRVMs. Our results showed there was no significantly difference in CPT1a expression by PHB2 ablation in NRVMs (Fig. S4). In addition, FAU was found to be strongly inhibited in NRVMs treated with siPHB2 (Fig. S5).

Taken together, in isolated NRVMs, PHB2 knockdowninduced cardiac lipid accumulation is consistent with that in isolated adult ventricular myocytes from $\mathrm{Phb2}$ cKO mice, which is shown by $\mathrm{LD}$ accumulation, reduced FAO and FAU. As CPT1b is downregulated by PHB2 deficiency in heart tissues and NRVMs, we suppose that PHB2 ablation impairs cardiac FAO in part by coordinating with decreased CPT1b.

\section{Impaired cardiac FAO is alleviated by overexpression of CPT1b in PHB2-deficient NRVMs}

Since PHB2 deficiency resulted in impaired cardiac FAO via downregulation of CPT1b, we hypothesized that upregulated expression of CPT1b could alleviate cardiac lipid accumulation.

In si-PHB2 NRVMs, CPT1b siRNA treatment promoted LD accumulation (Fig. 6a). Then we overexpressed CPT1b using adenoviruses in si-PHB2 NRVMs, and the expression of CPT1b and PHB2 was validated by western blot analysis (Fig. 6b). In si-PHB2 NRVMs treated with Ad-CPT1b, LD accumulation was obviously alleviated compared with si-PHB2 and Ad-Control group, reflected in decreased LD number, area and lower fluorescence intensity per NRVM (Fig. 6c). Moreover, FAO of BSAPalmitate group treated with (si-PHB2 + Ad-CPT1b) was much higher than that treated with (si-PHB2 + Ad-Control) (Fig. 6d). Meanwhile, FAO of BSA-Palmitate group treated with $(\mathrm{NC}+\mathrm{Ad}-\mathrm{CPT} 1 \mathrm{~b})$ was also higher than that treated with (NC + Ad-Control) (Fig. S6). In addition, FAU was not affected by overexpressing CPT1b in NC and si-PHB2 groups (Fig. 6e). Thus, CPT1b alleviated cardiac LD accumulation mainly via upregulating FAO in si-PHB2 NRVMs, rather than affecting FAU. Here, it should be noted that CPT1b overexpression only partially rescued the impairment of FAO caused by PHB2 deficiency, which indicated PHB2 deficiency might play a more dominant or complex role in downregulating FAO.

From the above study, we show that PHB2 plays a critical role in cardiac fatty acid metabolism. Ablation of PHB2 causes heart failure mainly through cardiac FAO disturbance and mitochondrial dysfunction. 
A

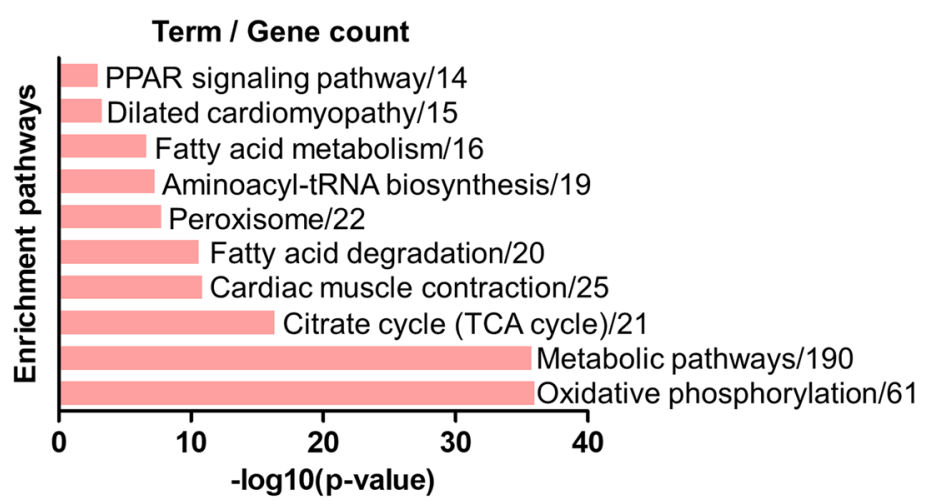

C

ETC / ATP synthesis

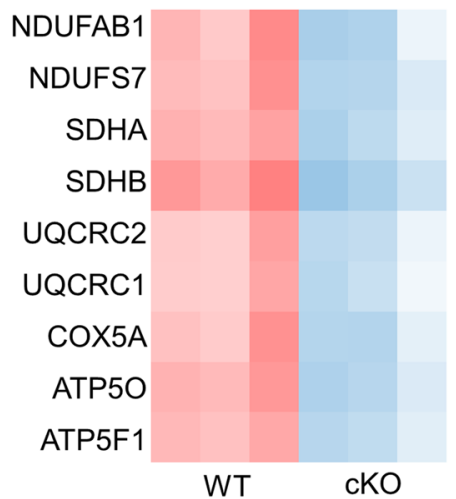

Fold change

$0.5 \quad 1.5$
D

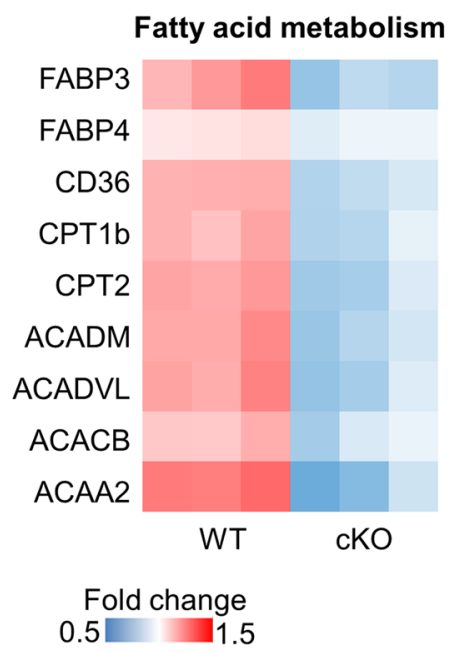

B

DCM / Cardiac muscle contraction

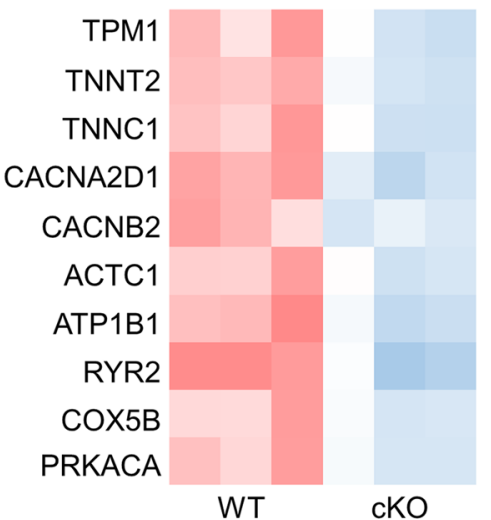

Fold change

$0.5 \quad 1.5$

E
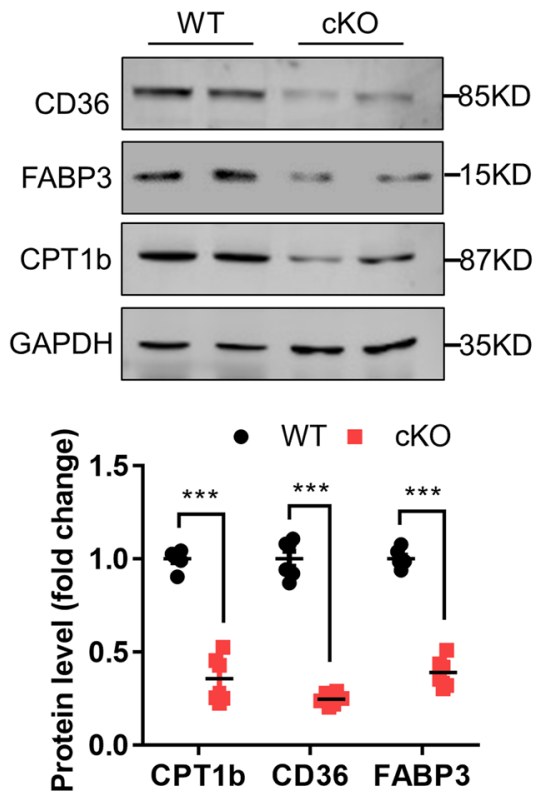

Fig. 4 Proteomic profiling of WT and Phb2 cKO mouse hearts. a KEGG pathway analysis of differentially expressed proteins from WT and Phb2 cKO mouse hearts at 6 weeks of age. b Heatmap showing downregulated proteins in the terms "Dilated cardiomyopathy" and "Cardiac muscle contraction" from KEGG pathway analysis in a. c Heatmap showing downregulated proteins in electron transport chain and ATP synthesis pathways from Phb2 cKO mouse hearts versus WT. d Heatmap of significantly decreased proteins in lipid metabolism from Phb2 cKO mouse hearts versus WT. e Western blot analysis of lipid metabolism proteins (top), including CD36, FABP3 and CPT1 b from WT and Phb2 cKO mouse hearts at 6 weeks of age. Anti-GAPDH immunoblot is used as the reference. Statistics of western blot (bottom), $n=3$ independent experiments per group. All Data represent mean \pm SEM. Significance was determined by two-tailed, unpaired Student's $t$ test. ns: no significance. ${ }^{* * *} p<0.001$ versus WT group.

\section{Discussion}

PHB2 deficiency causes heart failure and lethality in mice

Heart failure is one of the leading causes of death worldwide, which is characterized by perturbations in energy production and lack of adequate oxygen supply for maintaining proper heart contractility ${ }^{36}$. As deprivation of cardiac energy plays a major role in heart failure, the modulation of cardiac metabolism has promise as a target for the treatment of heart failure ${ }^{37,38}$.

Prohibitins (PHBs) are highly conserved proteins, which have been involved in multiple physiological and pathological processes. Prohibitins (prohibitin 1 and prohibitin 2) have been shown to play critical roles in aging, cancer, obesity, and mitophagy $8,14,39,40$. However, the function of 
A

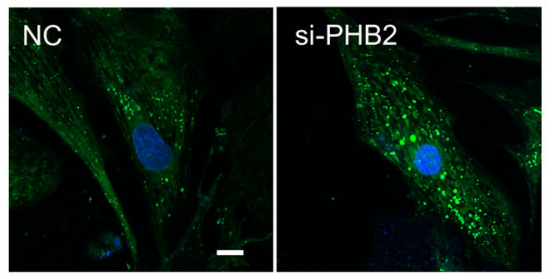

B

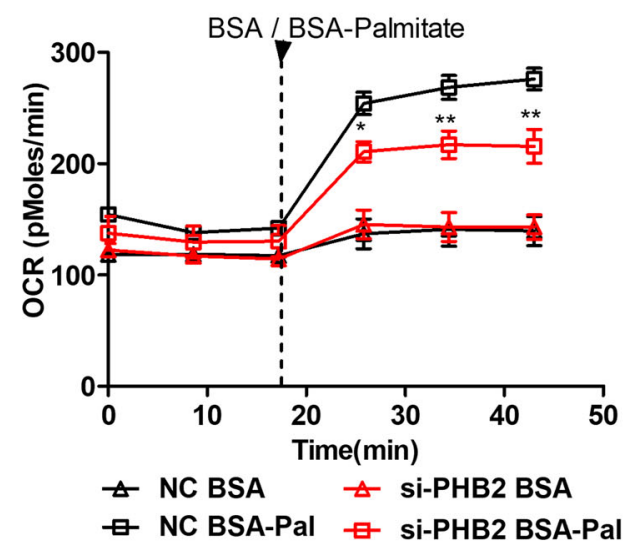

- WT $\because \mathrm{cKO}$
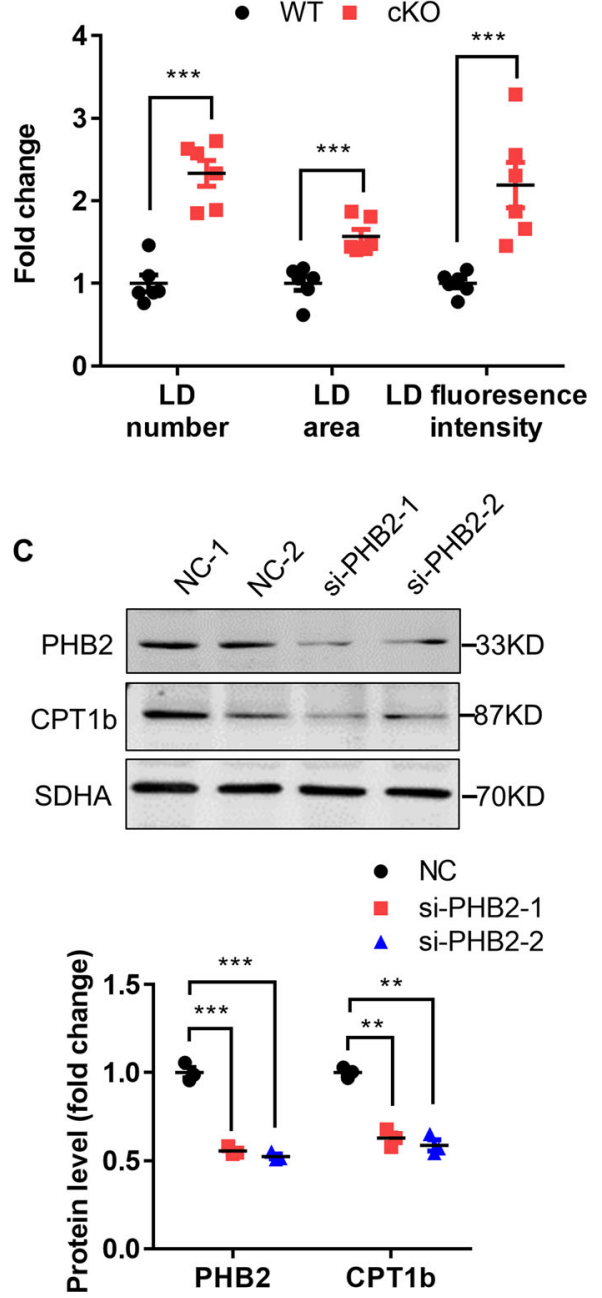

Fig. 5 Impaired cardiac FAO in PHB2-deficient neonatal rat ventricular myocytes (NRVMs). a Representative confocal images of LD visualized by BODIPYTM 493/503 in NRVMs treated with NC or si-PHB2 (left). Statistics of fold change of LD number, area and fluorescence intensity per NRVM (right). $n=100-120$ cells from eight independent experiments per group. Scale bar: $10 \mu \mathrm{m}$. b Measurement of FAO in NRVMs treated with NC or siPHB2. Arrowhead indicates the time points of adding BSA $(0.17 \mathrm{mM})$ or BSA-palmitate $(1 \mathrm{mM}) . n=3$ independent experiments per group. $\mathbf{c}$ Western blot analysis of PHB2 and CPT1 b in NRVMs treated with NC or si-PHB2 (top). Anti-SDHA immunoblot is used as the reference. Two siRNAs were used to target PHB2 (si-PHB2-1 and si-PHB2-2). Statistics of WB (bottom). $n=3$ independent experiments per group. All Data represent mean \pm SEM. Significance was determined by two-tailed, unpaired Student's $t$ test. ns: no significance. ${ }^{*} p<0.05,{ }^{* *} p<0.01,{ }^{* * *} p<0.001$ versus NC group. NC: negative control.

cardiac PHB2 is still largely unknown. In our study, by generating cardiac specific Phb2 knockout mice, we demonstrate that PHB2 deficiency significantly impairs cardiac fatty acid oxidation (FAO) and leads to mitochondrial dysfunction, and eventually results in severe heart failure at an earlier age.

\section{PHB2 is a potential inner mitochondrial membrane FAO regulator}

Cardiac-specific Phb2 knockout mice exhibited severe heart failure shown by dilated left ventricles, interstitial fibrosis, and systolic dysfunction. Accumulation of cardiac lipid droplets (LD) and mitochondrial metabolic dysfunction were also detected in $P h b 2$ cKO hearts. Thus, our study indicated that PHB2 is critical for maintaining the homeostasis of cardiac lipid metabolism.

As we know, the preferentially utilized substrate is fatty acid in healthy cardiac muscle. In a fasting state, myocardial fatty acid oxidation provides almost $70 \%$ of cardiac $\mathrm{ATP}^{41}$. Impaired FAO has been shown to play a key role in multiple mechanisms of heart failure ${ }^{41,42}$. In other organs, PHBs have been shown to be involved in lipid metabolism. For example, in Mito-Ob mice, PHBs have been shown to be involved in metabolic regulation of adipose tissue homeostasis ${ }^{39}$. Another study discovered loss of PHB2/DNAJC19 complexes affects cardiolipin 

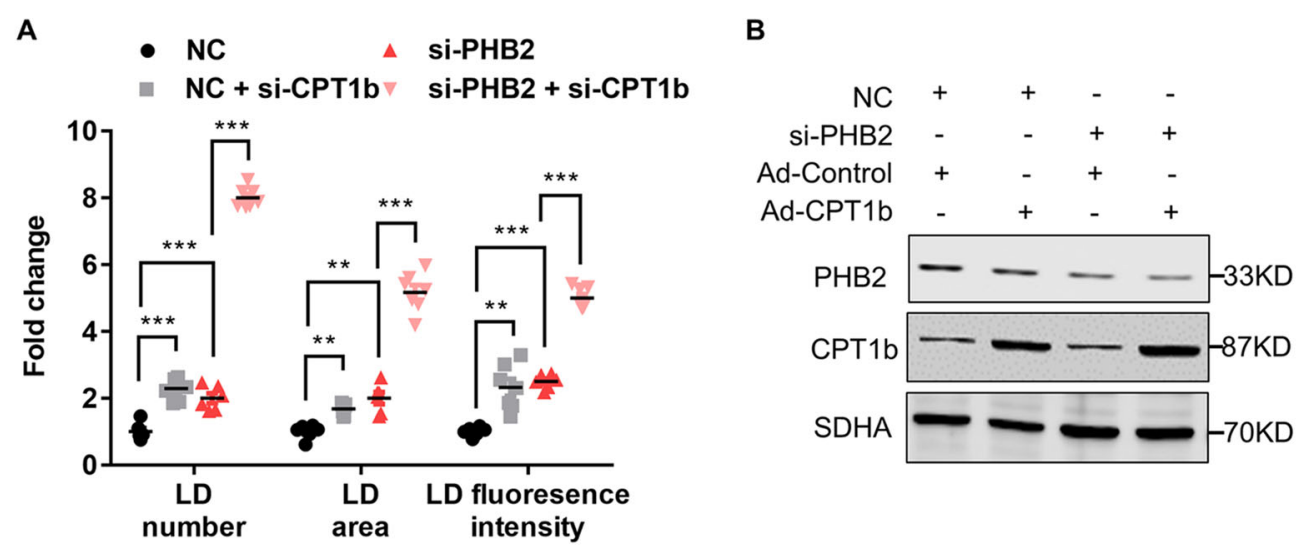

C
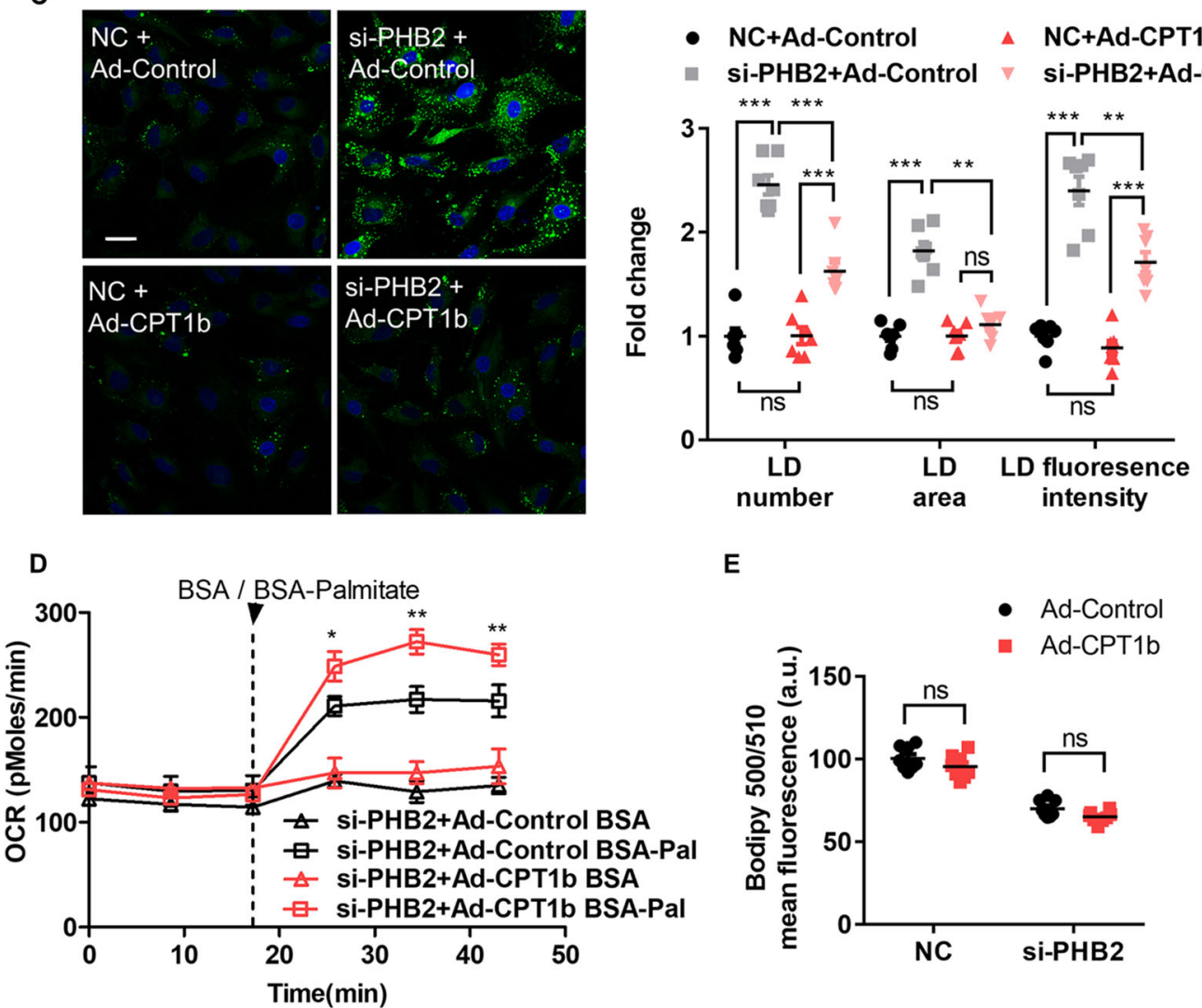

E

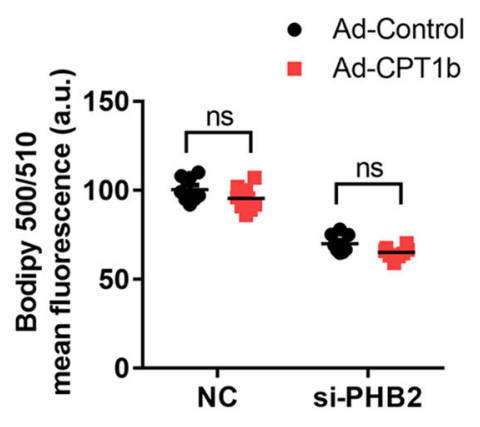

Fig. 6 Upregulation of carnitine palmitoyltransferase1b (CPT1b) alleviates cardiac FAO disturbance in PHB2-deficient NRVMs. a Measurement of LD visualized by BODIPYTM 493/503 in NRVMs treated with NC, si-PHB2, NC + si-CPT1b, or si-PHB2 + si-CPT1b, respectively. $n=$ 100-120 cells from seven independent experiments per group. b Western blot analysis of PHB2 and CPT1b in NRVMs treated with NC + Ad-Control, $\mathrm{NC}+\mathrm{Ad}-\mathrm{CPT} 1 \mathrm{~b}, \mathrm{si}-\mathrm{PHB} 2+\mathrm{Ad}-\mathrm{Control}$, or si-PHB2 + Ad-CPT1b, respectively. Anti-SDHA immunoblot is used as the reference. $\mathbf{c}$ Representative confocal images of LD visualized by BODIPYTM 493/503 in NRVMs treated with NC + Ad-Control, NC + Ad-CPT1b, si-PHB2 + Ad-Control, or si-PHB2 + Ad-CPT1b, respectively (left). Statistics of fold change of LD number, area and fluorescence intensity per NRVM (right). $n=100-120$ cells from six independent experiments per group. Scale bar: $20 \mu \mathrm{m}$. d Measurement of FAO in NRVMs treated with si-PHB2 + Ad-Control, or si-PHB2 + Ad-CPT1b, respectively. Arrowhead indicates the time point when BSA $(0.17 \mathrm{mM})$ or BSA-palmitate $(1 \mathrm{mM})$ was added. $n=3$ independent experiments per group. e Measurement of FAU visualized by BODIPYTM 500/510 in NRVMs treated with NC + Ad-Control, NC + Ad-CPT1b, si-PHB2 + Ad-Control or siPHB2 + Ad-CPT1b, respectively. $n=100-120$ cells from seven independent experiments per group. All Data represent mean \pm SEM. Significance was determined by two-tailed, unpaired Student's $t$ test. ns: no significance. ${ }^{*} p<0.05,{ }^{* *} p<0.01,{ }^{* * *} p<0.001$ versus control group. 
acylation and leads to the accumulation of cardiolipin species $^{43}$. However, the functions of PHB2 in cardiac fatty acid metabolism have not yet been reported.

In our study, PHB2 was identified as a potential FAO regulator required for homeostasis of cardiac fatty acid metabolism. In isolated cardiomyocytes from $\mathrm{Phb2} \mathrm{cKO}$ mice and si-PHB2 NRVMs, large numbers of LD significantly accumulated, shown by higher LD number, area and higher fluorescence intensity. More importantly, PHB2 deficiency resulted in decreased CPT1b, and upregulated expression of CPT1b partially alleviated cardiac lipid accumulation caused by PHB2 deficiency. In summary, cardiac PHB2 deficiency-induced mitochondrial dysfunction resulted in fatty acid metabolic disorder through downregulating CPT1b, which played a major role in the course of heart failure in Phb2 cKO mice. Here, different from increased body weight in obesity or diabetes mouse models with lipotoxic cardiomyopathy, there was no difference in body weight between WT and Phb2 cKO mice. Therefore, it suggested that PHB2 may play a unique role in modulating cardiac fatty acid metabolism.

\section{Studying PHB2 signaling will contribute to a novel treatment strategy for heart failure}

The pathogenesis of heart failure is very complex. As we described above, to elucidate the mechanisms for modulating homeostasis of cardiac metabolism will greatly help to develop promising treatments for heart failure. In our study, we found that PHB2 deficiency in the heart impaired cardiac FAO and reduced ATP level, and that these defects in Phb2 cKO hearts occurred at 6 weeks of age, prior to the occurrence of heart failure which was evident at 8 weeks of age. Therefore, our study provided the evidence for causal relationships between FAO impairment and heart failure. Meanwhile, our study revealed the connection between PHB2 and $\mathrm{CPT} 1 \mathrm{~b}$ in cardiac fatty acid oxidation and upregulation of CPT1b that could partially alleviate lipid accumulation caused by PHB2 deficiency. Thus, our study indicated that CPT1b might be a potential therapeutic target for heart failure. However, it is controversial with current therapy for acute heart failure using CPT1b inhibitor $^{16,41}$. The ablation of cardiac PHB2 exhibits a more severe heart failure at an early age $(50 \%$ mortality at day 70); however, haploinsufficiency of CPT1b mice showed heart failure only under a severe pressure-overload condition. Thus, decreased cardiac FAO might partially account for heart failure. In our study, mitochondrial dysfunction shown by profound ultrastructural alteration in cristae morphology, elevated ROS, reduced mitochondrial membrane potential and decreased ATP content were found in Phb2 cKO mouse hearts. We supposed that ablation of PHB2 might trigger additional stress to intensify cardiac dysfunction.
In addition, accumulating evidences show that autophagy is critical in metabolic homeostasis maintenance, protein quality control and mitochondrial network remodeling, which offers autophagy as an emerging target for treating cardiorenal metabolic disease ${ }^{4}$. Here, as PHB2 is an inner mitochondrial membrane mitophagy receptor $^{14}$ and autophagy is reported to be involved in disturbed lipid metabolism ${ }^{44-46}$, the roles of PHB2 on cardiac autophagy in heart failure or other cardiac pathologies should be further investigated in future studies.

Taken together, our findings provide direct evidence that PHB2 plays an essential role in cardiac fatty acid metabolism homeostasis and also demonstrate that CPT1b is downstream of PHB2 signaling pathway. Phb2 cKO mice exhibited severe heart failure and died at an average age 10 weeks, with cardiac lipid accumulation and mitochondrial dysfunction. Thus, our study points to the distinct possibility that PHB2 is an inner mitochondrial membrane FAO regulator. Further studies on PHB2's control of mitochondrial metabolic homeostasis will contribute to develop or optimize therapeutic approaches to heart failure.

\section{Materials and methods \\ Animal care}

All animal experiments were carried out according to the rules of the American Association for the Accreditation of Laboratory Animal Care International and the Guide for the Care and Use of Laboratory Animals published by the US National Institutes of Health (NIH Publication No. 85-23, revised 1996). All procedures were approved by the Animal Care Committee of Peking University accredited by AAALAC International.

\section{Reagents}

Dulbecco's modified Eagle's medium (DMEM), Lipofectamine RNAiMax, penicillin and streptomycin were from Invitrogen (Carlsbad, CA). Fetal bovine serum (FBS) was from Gibco (Carlsbad, CA). MitoSOX, DCF, TMRM, BODIPY $^{\mathrm{TM}}$ 500/510, BODIPY ${ }^{\mathrm{TM}}$ 493/503 were from Molecular Probes (Eugene, OR). Mycoplasma contamination was detected using Mycoplasma Detection Kit (Beyotime, China).

\section{Strategy of generating cardiac-specific Phb2 knockout mice}

The cardiac-specific knockout mice were generated as previously described ${ }^{47}$. Floxed Phb2 mice were obtained from the Nanjing Biomedical Research Institute of Nanjing University. Exon4 of the Phb2 gene was inserted into two flanking LoxP sites. Homozygous Phb2-floxed mice $\left(P h b 2^{\text {flox/flox }}\right)$ were obtained by inbreeding of the $P h b 2^{\text {flox/+ }}$ mice, which were generated from C57BL/6 J 
mice by the CRISPR/Cas9 system. Briefly, $P h b 2^{\text {flox/flox }}$ mice were bred with Mlc2v-Cre mice in which Cre recombinase expression was controlled by the myosin light chain $2 \mathrm{v}$ promoter to generate double heterozygous Mlc2v-Cre and Phb2 floxed mice (Mlc2v-Cre ${ }^{+}$and $\left.P h b 2^{\mathrm{flox} /+}\right)$. The mice were then backcrossed with homozygous $\mathrm{Phb} 2^{\text {flox/flox }}$ mice to generate $\mathrm{Mlc} 2 \mathrm{v}-\mathrm{Cre}^{+}$ and $P h b 2^{\text {flox/flox }}$ as cardiac-specific Phb2 knockout mice, Mlc2v-Cre ${ }^{-}$and $P h b 2^{\text {flox/flox }}$ mice as littermate controls.

Mice were genotyped by PCR using mouse tail DNA, and flox primers (forward, TGCTCTGGAGAAAGTGC CCC; reverse, CACACACCACAAACAGCAACAG) and Cre primers (forward, GCGGTCTGGCAGTAAAAACT ATC; reverse, GTGAAACAGCATTGCTGTCACTT), respectively. PCR program: $95^{\circ} \mathrm{C}, 10 \mathrm{~min} ; 95^{\circ} \mathrm{C}, 30 \mathrm{~s}$, $52^{\circ} \mathrm{C}, 30 \mathrm{~s}, 72^{\circ} \mathrm{C}, 45 \mathrm{~s}$ ( 35 cycles); $72^{\circ} \mathrm{C}, 5 \mathrm{~min} ; 4^{\circ} \mathrm{C}$, hold.

\section{Histology and immunohistochemistry}

The hearts were perfused with $2.5 \%$ glutaraldehyde and $4 \%$ paraformaldehyde at 8 weeks of age. Then the hearts were fixed in $4 \%$ paraformaldehyde overnight, bisected transversely at the mid-ventricular level, embedded in paraffin and cut into $4 \mathrm{~mm}$ sections for staining with hematoxylin and eosin (HE). Additionally, heart sections were stained with Masson's trichrome for fibrosis analysis. Oil Red O staining on frozen sections was performed to examine lipid accumulation in hearts. Eight regions were randomly assigned from the full-size crosscut heart for quantification of Masson staining, each consisting of a rectangle of $515 \times 434 \mu^{2}$. Image J was used for fibrosis areas quantification.

\section{Echocardiography}

Male mice were randomly chosen to be anesthetized with isoflurane, and performed transthoracic echocardiography using a VEVO-2100 Imaging System (Visual Sonics) at 4, 6, 8 weeks of age, respectively. The group allocations for echocardiography were reviewed blindly by the investigator. Fractional shortening (FS) and ejection fraction $(\mathrm{EF})$ were used to assess systolic function. Left ventricular posterior wall thickness at end-diastole and end-systole (LVPW; d and LVPW; s), left ventricular internal diameter at end-diastole and end-systole (LVID; $\mathrm{d}$ and LVID; s), left ventricular volume at end-diastole and end-systole (LV Vol; d and LV Vol; s) were obtained from the M-mode image.

\section{Isolation of adult mouse cardiomyocytes}

Single ventricular myocytes were isolated from the hearts of randomly chosen wild type, Phb2 cardiacspecific knockout mice at 6 weeks of age, as previously described $^{48-50}$. Freshly-isolated cardiomyocytes were plated on culture dishes covered with laminin (Sigma) for at least $1 \mathrm{~h}$ and then the attached cells were in DMEM medium (Invitrogen, Carksbad, California) along with 10\% FBS (Gibco), $5 \mathrm{mM}$ BDM (Sigma), and 1\% InsulinTransferrin-Selenium (ITS) Supplement (Invitrogen) until use.

Isolation and culture of neonatal rat ventricular myocytes Ventricular myocytes were isolated from 1-day-old Sprague-Dawley rats, as previously described ${ }^{51}$. Neonatal rat ventricular myocytes (NRVMs) were plated at $1.5 \times$ $10^{5}$ cells $/ \mathrm{cm}^{2}$ in DMEM supplemented with $10 \%$ FBS and $1 \%$ penicillin and streptomycin in the presence of $0.1 \mathrm{mM}$ 5-bromo-2-deoxyuridine (Sigma). Adenovirus infection or siRNA transfection was implemented after $24 \mathrm{~h}$ quiescence in serum-free DMEM following 48-72 $\mathrm{h}$ culture in DMEM containing 10\% FBS.

\section{Cell culture, siRNA transfection and adenovirus infection}

For RNA interference, the negative control was used as control for all siRNA experiments. $100 \mathrm{nM}$ siRNA were transiently transfected into the NRVMs with RNAi Max according to the manufacturers' instructions. The siRNAs used in this study were listed (Table S2).

For adenovirus infection, NRVMs were infected with adenovirus carrying the $\mathrm{C}$ terminal Myc-tagged $\mathrm{CPT} 1 \mathrm{~b}$ gene at a multiplicity of infection (m.o.i.) of 20. Confocal imaging and western blot analysis were performed $72 \mathrm{~h}$ after siRNA transfection and $48 \mathrm{~h}$ after virus infection.

\section{Confocal microscopy and image processing}

Zeiss LSM710 inverted confocal microscope with a $40 \times$ 1.3 NA oil-immersion objective was used for acquiring images. All experiments were carried out with Tyrode's solution $(137 \mathrm{mM} \mathrm{NaCl}, 5.4 \mathrm{mM} \mathrm{KCl}, 1.2 \mathrm{mM} \mathrm{MgCl} 2$, $1.2 \mathrm{mM}$ NaH2PO4, $1.8 \mathrm{mM} \mathrm{CaCl} 2,10 \mathrm{mM}$ glucose, and $20 \mathrm{mM}$ HEPES, $\mathrm{pH} 7.35$, adjusted with $\mathrm{NaOH}$ ) at room temperature $\left(22-26^{\circ} \mathrm{C}\right)$.

For measuring mitochondrial ROS, mitoSOX $(5 \mu \mathrm{M})$ was loaded into isolated cardiomyocytes in Tyrode's solution at $37^{\circ} \mathrm{C}$ for $10 \mathrm{~min}$ and washed three times before imaging. Fluorescence of mitoSOX was taken by excitation at $514 \mathrm{~nm}$ and emission collection at $>500 \mathrm{~nm}$.

For measuring cytosolic ROS levels, DCF $(5 \mu \mathrm{M})$ was loaded into isolated cardiomyocytes in Tyrode's solution at $37^{\circ} \mathrm{C}$ for $20 \mathrm{~min}$ and washed three times before imaging. Fluorescence of DCF was taken by excitation at $488 \mathrm{~nm}$ and emission collection at $>500 \mathrm{~nm}$.

For measuring mitochondrial membrane potential, TMRM $(100 \mathrm{nM})$ was loaded into isolated cardiomyocytes in Tyrode's solution at $37^{\circ} \mathrm{C}$ for $10 \mathrm{~min}$. Fluorescence of TMRM was taken by excitation at $543 \mathrm{~nm}$ and emission collection at $>560 \mathrm{~nm}$.

For detecting fatty acids uptake, BODIPY ${ }^{\mathrm{TM}} 500 / 510$ $(20 \mu \mathrm{M})$ was loaded into isolated cardiomyocytes for 2 min in Tyrode's solution at $37^{\circ} \mathrm{C}$ and washed three 
times before imaging. BODIPY ${ }^{\mathrm{TM}} 500 / 510$ fluorescence were both taken by excitation at $488 \mathrm{~nm}$ and emission collection at $500-550 \mathrm{~nm}$.

For visualizing LD, BODIPY ${ }^{\mathrm{TM}} 493 / 503(20 \mu \mathrm{M})$ was loaded into isolated cardiomyocytes for $10 \mathrm{~min}$ in Tyrode's solution at $37^{\circ} \mathrm{C}$ and washed three times before imaging. BODIPY ${ }^{\mathrm{TM}}$ 493/503 fluorescence were both taken by excitation at $488 \mathrm{~nm}$ and emission collection at $500-550 \mathrm{~nm}$.

Confocal images were analyzed using Interactive Data Language (IDL, Research Systems) software and customer-devised programs.

\section{Western blot}

Heart tissues or isolated cardiomyocytes were homogenized and lysed in lysis buffer (30 mM HEPES, $100 \mathrm{mM}$ $\mathrm{NaCl}, 0.5 \%$ Nonidet P (NP)-40, protease inhibitors mixture, $\mathrm{pH}$ 7.6) on ice for $10 \mathrm{~min}$, and the lysates were centrifuged at 13,000 rpm for $20 \mathrm{~min}$. Cell lysates $(50 \mu \mathrm{g} /$ line) were separated by $12 \%$ SDS-PAGE and transferred to PVDF $0.2 \mathrm{~mm}$ membranes. Membranes were blotted with $5 \%$ dry, non-fat milk prepared in Tris-buffer saline-plus $0.1 \%$ Tween-20 (TBST) at room temperature for one hour and incubated with primary antibodies diluted in 5\% milk overnight at $4{ }^{\circ} \mathrm{C}$. Blots were visualized using secondary antibodies conjugated with IRDye (LI-COR) by an Odyssey imaging system (LI-COR).

The following antibodies were used for western blotting: PHB2 (1:1000, CST, 14085), SDHA (1:2000, Abcam, ab14715), CPT1b (1:1000, Abcam, ab134988), TIM23 (1:2000, BD Transduction Laboratories ${ }^{\mathrm{TM}}$, 611222), GAPDH (1:2000, ABclonal, AC002), CD36 (1:500, Thermo Fisher, PA1-16813), FABP3 (1:1000, Proteintech, 10676-1-AP), CPT1a (1:1000, Proteintech, 15184-1-AP).

\section{Transmission electron microscopy}

Fresh excised hearts from 6-week-old mice were perfused by $1 \%$ glutaraldehyde and $4 \%$ paraformaldehyde for $30 \mathrm{~min}$. After dissecting into $1-2 \mathrm{~mm}^{3}$ blocks, samples were immediately fixed with $2.5 \%$ glutaraldehyde and $4 \%$ paraformaldehyde, then post-fixed with $1 \%$ osmium tetroxide $/ 1.5 \%$ potassium ferrocyanide. Following several washes in distilled water, samples were stained with $2 \%$ aqueous uranyl acetate for $2 \mathrm{~h}$ at room temperature. After rinsing several times in distilled water, the specimens were dehydrated through a graded alcohol series and subsequently embedded in Spurr's resin (SPI supplies, PA, USA). Ultra-thin sections $(70 \mathrm{~nm})$ were cut with a diamond knife using an ultramicrotome (UC7, Leica Microsystem), and collected on copper grids with a single slot. After counterstaining with uranyl acetate and lead citrate, sections were examined using an electron microscopy (Tecnai G2 20 TWIN, FEI) at $120 \mathrm{kV}$.

\section{Measurement of mitochondrial respiratory function}

The mitochondrial respiration was measured using XF24 Extracellular Flux Analyzer (Seahorse Bioscience, North Billerica, MA) according to the manufacturer's instructions. Briefly, isolated cardiomyocytes were cultured in XF24 cell-culture microplates at $10^{5}$ cells/well in DMEM supplemented with 10\% FBS. After adherence, the culture medium was changed to assay medium (Sigma D5030) supplemented with $2 \mathrm{mM}$ GlutaMAX (Gibco), $2.5 \mathrm{mM}$ sodium pyruvate, and $25 \mathrm{mM}$ glucose $(\mathrm{pH} 7.4$ at $37^{\circ} \mathrm{C}$ ). Then cardiomyocytes were incubated for $1 \mathrm{~h}$ at $37^{\circ} \mathrm{C}$ prior to measurement. Bioenergetic analyses were performed in an XF24 Extracellular Flux Analyzer with oligomycin $(1 \mu \mathrm{M})$, FCCP $(1 \mu \mathrm{M})$, rotenone $(1 \mu \mathrm{M})$, and antimycin A (AA, $1 \mu \mathrm{M})$ injected sequentially. After each drug injection, the OCR was measured three times. Basal OCR refers to the respiration rate measured prior to the addition of oligomycin. Maximal OCR was calculated by subtracting the OCR in the presence of rotenone and AA from that in the presence of FCCP.

\section{Measurement of mitochondrial fatty acid oxidation}

Isolated cardiomyocytes were cultured in XF24 cellculture microplates at 300 cells/well in DMEM supplemented with $10 \%$ FBS. After adherence, the culture medium was changed to Krebs-Henseleit Buffer (KHB component: $\mathrm{NaCl} 111 \mathrm{mM}, \mathrm{KCl} 4.7 \mathrm{mM}, \mathrm{MgSO} 42 \mathrm{mM}$, Na2HPO4 $1.2 \mathrm{mM}$, Glucose $2.5 \mathrm{mM}$, Carnitine $0.5 \mathrm{mM}$ ), and cardiomyocytes were incubated for $1 \mathrm{~h}$ at $37^{\circ} \mathrm{C}$ prior to measurement. $1 \mathrm{mM}$ BSA-Palmitate solution and $0.17 \mathrm{mM}$ BSA solution were prepared according to XF BSA-Palmitate FAO Reagent instructions. BSA-Palmitate or BSA was injected at the time points. Mitochondrial fatty acid oxidation was calculated by subtracting the OCR in the presence of BSA from that in the presence of BSA-Palmitate.

\section{ATP measurement}

The ATP concentration in heart was measured with the Enlighten ATP assay system using luciferase and luciferin (Promega). Briefly, the hearts were excised and lysed with Tris-phenol. The myocardial ATP content was measured with the luciferase assay according to manufacturer's instructions.

\section{Quantitative proteomic analysis by tandem mass tag (TMT) technology}

For each sample (cKO VS WT), $20 \mu \mathrm{g}$ of proteins were mixed with $5 \times$ loading buffer and boiled for $5 \mathrm{~min}$. The proteins were then separated on $12.5 \%$ SDS-PAGE gel (constant current $14 \mathrm{~mA}, 90 \mathrm{~min}$ ) and protein bands were visualized by Coomassie Blue R-250 staining for quality control. After that, $200 \mu \mathrm{g}$ of proteins from each sample 
were prepared essentially as previously described with filter-aided sample preparation (FASP Digestion) ${ }^{52}$.

Using TMT reagents according to the manufacturer's instructions, $100 \mu \mathrm{g}$ of the peptide mixture from each sample was labeled (Thermo Scientific, Massachusetts, USA). A Pierce high $\mathrm{pH}$ reverse-phase fractionation kit (Thermo Scientific) was used to fractionate TMT-labeled digested samples into 15 fractions by an increasing acetonitrile stepgradient elution carried according to the instructions.

Liquid chromatography-mass spectrometry/MS (LCMS/MS) analysis was performed on a $Q$ Exactive mass spectrometer (Thermo Scientific) that was coupled to Easy nLC for $60 \mathrm{~min}$. The mass spectrometer was operated in positive ion mode. Tandem mass spectrometry (MS/MS) spectra were searched using MASCOT engine (Matrix Science, London, UK; version 2.2) embedded into Proteome Discoverer 1.4.

The differentially expressed proteins were identified by fold change values of greater than \pm 1.2 and $p<0.05$ (from Mann-Whitney U-tests). The KEGG pathway enrichment analysis for both upregulated and downregulated genes were performed using Database for Annotation, Visualization, and Integrated Discovery ${ }^{53}$ with a P-value cutoff of 0.05 under the Benjamini test.

\section{Statistics}

The data are presented as mean \pm SEM, and two-tailed Student's $t$ test was applied to determine statistical significance. $P<0.05$ was considered statistically significant. The log-rank test was used for survival curves. We did not use any specific test to estimate statistical power. Sample size was chosen based on the previous experience obtained from the study of mouse models or biological measurements. The number of replications is listed in each figure legend.

\footnotetext{
Acknowledgements

The authors thank Ms. Yao Song and Yuli Liu for echocardiography, Ms. Dongwei Ma and Xueting Sun for histology, Ms. Wenwen Li and Fengli Xu for heart perfusion, Dr. Ying-Chun Hu, Yun-Chao Xie and Hong-Mei Zhang for their professional technical assistance in EM sample preparation and image analysis, Mr. Tao Sun, Jinghang Li and Dapeng Zhang for data analysis, and Drs Ruiping Xiao, Xiaowei Chen, Xiaotao Duan and Yan Zhang for valuable comments. This work was supported by the National Key Basic Research Program of China (2017YFA0504000 and 2016YFA0500403), the National Science Foundation of China (31670039, 8182780030, 31821091, 31470811, 81670894, and 81721003), State Key Laboratory of Ophthalmology (Zhongshan Ophthalmic Center, Sun Yat-Sen University), Pearl River Talents Program-Local Innovative and Research Teams (2017BT01S138), "100 talents plan" from Sun Yat-sen University, the Open Research Funds of the State Key Laboratory of Ophthalmology (2017KF05) and Funds of the Guangdong Provincial Key Laboratory of Ophthalmology and Visual Science (2017B030314025), Guangzhou, Guangdong Province, China
}

Conflict of interest

The authors declare that they have no conflict of interest.

\section{Publisher's note}

Springer Nature remains neutral with regard to jurisdictional claims in published maps and institutional affiliations.

Supplementary Information accompanies this paper at (https://doi.org/ 10.1038/s41419-020-2374-7).

Received: 28 October 2019 Revised: 24 February 2020 Accepted: 25 February 2020

Published online: 12 March 2020

\section{References}

1. Bui, A. L., Horwich, T. B. \& Fonarow, G. C. Epidemiology and risk profile of heart failure. Nat. Rev. Cardiol. 8, 30-41 (2011).

2. Doenst, T., Nguyen, T. D. \& Abel, E. D. Cardiac metabolism in heart failure: implications beyond ATP production. Circulation Res. 113, 709-724 (2013).

3. Ren, J. \& Zhang, Y. Emerging therapeutic potential targeting genetics and epigentics in heart failure. Biochim. Biophys. Acta Mol. Basis Dis. 1863, 1867-1869 (2017).

4. Zhang, Y., Whaley-Connell, A. T., Sowers, J. R. \& Ren, J. Autophagy as an emerging target in cardiorenal metabolic disease: from pathophysiology to management. Pharmacol. Therapeutics 191, 1-22 (2018).

5. Lampert, M. A. et al. BNIP3L/NIX and FUNDC1-mediated mitophagy is required for mitochondrial network remodeling during cardiac progenitor cell differentiation. Autophagy 15, 1182-1198 (2019).

6. Palaniyandi, S. S., Qi, X., Yogalingam, G., Ferreira, J. C. \& Mochly-Rosen, D. Regulation of mitochondrial processes: a target for heart failure. Drug Discov. Today Dis. Mech. 7, e95-e102 (2010).

7. Dey, S., DeMazumder, D., Sidor, A., Foster, D. B. \& O'Rourke, B. Mitochondrial ROS drive sudden cardiac death and chronic proteome remodeling in heart failure. Circulation Res. 123, 356-371 (2018).

8. Artal-Sanz, M. \& Tavernarakis, N. Prohibitin couples diapause signalling to mitochondrial metabolism during ageing in C. elegans. Nature 461, 793-797 (2009).

9. Tatsuta, T., Model, K. \& Langer, T. Formation of membrane-bound ring complexes by prohibitins in mitochondria. Mol. Biol. cell 16, 248-259 (2005).

10. Nijtmans, L. G., Artal, S. M., Grivell, L. A. \& Coates, P. J. The mitochondrial PHB complex: roles in mitochondrial respiratory complex assembly, ageing and degenerative disease. Cell. Mol. life Sci.: CMLS 59, 143-155 (2002).

11. Mishra, S., Murphy, L. C. \& Murphy, L. J. The Prohibitins: emerging roles in diverse functions. J. Cell. Mol. Med. 10, 353-363 (2006).

12. Osman, C., Merkwirth, C. \& Langer, T. Prohibitins and the functional compartmentalization of mitochondrial membranes. J. Cell Sci. 122, 3823-3830 (2009).

13. Jian, C. et al. Deficiency of PHB complex impairs respiratory supercomplex formation and activates mitochondrial flashes. J. Cell Sci. 130, 2620-2630 (2017).

14. Wei, Y., Chiang, W. C., Sumpter, R. Jr., Mishra, P. \& Levine, B. Prohibitin 2 is an Inner Mitochondrial Membrane Mitophagy Receptor. Cell 168, 224-238.e210 (2017).

15. Yan, C. et al. PHB2 (prohibitin 2) promotes PINK1-PRKN/Parkin-dependent mitophagy by the PARL-PGAM5-PINK1 axis. Autophagy 16, 419-434 (2019).

16. Lopaschuk, G. D., Ussher, J. R., Folmes, C. D., Jaswal, J. S. \& Stanley, W. C. Myocardial fatty acid metabolism in health and disease. Physiol. Rev. 90, 207-258 (2010).

17. Stanley, W. C., Recchia, F. A. \& Lopaschuk, G. D. Myocardial substrate metabolism in the normal and failing heart. Physiol. Rev. 85, 1093-1129 (2005).

18. Welte, M. A. Expanding roles for lipid droplets. Curr. Biol.: CB 25, R470-R481 (2015).

19. Tian, Z. et al. ANGPTL2 activity in cardiac pathologies accelerates heart failure by perturbing cardiac function and energy metabolism. Nat. Commun. 7, 13016 (2016)

20. Stanley, W. C. \& Recchia, F. A. Lipotoxicity and the development of heart failure: moving from mouse to man. Cell Metab. 12, 555-556 (2010).

21. Brown, N. F., Weis, B. C., Husti, J. E., Foster, D. W. \& McGarry, J. D. Mitochondrial carnitine palmitoyltransferase I isoform switching in the developing rat heart. J. Biol. Chem. 270, 8952-8957 (1995).

22. Weis, B. C., Cowan, A. T., Brown, N., Foster, D. W. \& McGarry, J. D. Use of a selective inhibitor of liver carnitine palmitoyltransferase । (CPT I) allows quantification of its contribution to total CPT I activity in rat heart. Evidence 
that the dominant cardiac CPT I isoform is identical to the skeletal muscle enzyme. J. Biol. Chem. 269, 26443-26448 (1994).

23. He, L. et al. Carnitine palmitoyltransferase-1b deficiency aggravates pressure overload-induced cardiac hypertrophy caused by lipotoxicity. Circulation $\mathbf{1 2 6}$ 1705-1716 (2012).

24. Artal-Sanz, M. et al. The mitochondrial prohibitin complex is essential for embryonic viability and germline function in Caenorhabditis elegans. J. Biol. Chem. 278, 32091-32099 (2003).

25. Park, S. E. et al. Genetic deletion of the repressor of estrogen receptor activity (REA) enhances the response to estrogen in target tissues in vivo. Mol. Cell. Biol. 25, 1989-1999 (2005).

26. Li, L. et al. In vivo stabilization of OPA1 in hepatocytes potentiates mitochondrial respiration and gluconeogenesis in a prohibitin-dependent way. J. Biol. Chem. 294, 12581-12598 (2019).

27. Ising, C. et al. Prohibitin-2 depletion unravels extra-mitochondrial functions at the kidney filtration barrier. Am. J. Pathol. 186, 1128-1139 (2016).

28. Supale, S. et al. Loss of prohibitin induces mitochondrial damages altering beta-cell function and survival and is responsible for gradual diabetes development. Diabetes 62, 3488-3499 (2013).

29. Merkwirth, C. et al. Loss of prohibitin membrane scaffolds impairs mitochondrial architecture and leads to tau hyperphosphorylation and neurodegeneration. PLoS Genet. 8, e1003021 (2012).

30. Civiletto, G. et al. Opa1 overexpression ameliorates the phenotype of two mitochondrial disease mouse models. Cell Metab. 21, 845-854 (2015).

31. Goldberg, I. J., Trent, C. M. \& Schulze, P. C. Lipid metabolism and toxicity in the heart. Cell Metab. 15, 805-812 (2012).

32. Gocze, P. M. \& Freeman, D. A. Factors underlying the variability of lipid droplet fluorescence in MA-10 Leydig tumor cells. Cytometry 17, 151-158 (1994).

33. DiDonato, D. \& Brasaemle, D. L. Fixation methods for the study of lipid droplets by immunofluorescence microscopy. J. Histochem. Cytochem.: Off. J. Histochem. Soc. 51, 773-780 (2003).

34. Milger, $\mathrm{K}$. et al. Cellular uptake of fatty acids driven by the ER-localized acylCoA synthetase FATP4. J. Cell Sci. 119, 4678-4688 (2006).

35. Sorokina, N. et al. Recruitment of compensatory pathways to sustain oxidative flux with reduced carnitine palmitoyltransferase I activity characterizes inefficiency in energy metabolism in hypertrophied hearts. Circulation 115, 2033-2041 (2007)

36. Giordano, F. J. Oxygen, oxidative stress, hypoxia, and heart failure. J. Clin. Invest. 115, 500-508 (2005).

37. Palaniswamy, C., Mellana, W. M., Selvaraj, D. R. \& Mohan, D. Metabolic modulation: a new therapeutic target in treatment of heart failure. Am. J. Ther. 18 e197-e201 (2011).
38. Taegtmeyer, $\mathrm{H}$. Cardiac metabolism as a target for the treatment of heart failure. Circulation 110, 894-896 (2004).

39. Ande, S. R. et al. Prohibitin overexpression in adipocytes induces mitochondrial biogenesis, leads to obesity development, and affects glucose homeostasis in a sex-specific manner. Diabetes 63, 3734-3741 (2014).

40. Koushyar, S., Jiang, W. G. \& Dart, D. A. Unveiling the potential of prohibitin in cancer. Cancer Lett. 369, 316-322 (2015).

41. Lionetti, V., Stanley, W. C. \& Recchia, F. A. Modulating fatty acid oxidation in heart failure. Cardiovasc. Res. 90, 202-209 (2011).

42. Neglia, D. et al. Impaired myocardial metabolic reserve and substrate selection flexibility during stress in patients with idiopathic dilated cardiomyopathy. Am. J. Physiol. Heart Circ. Physiol. 293, H3270-H3278 (2007).

43. Richter-Dennerlein, R. et al. DNAJC19, a mitochondrial cochaperone associated with cardiomyopathy, forms a complex with prohibitins to regulate cardiolipin remodeling. Cell Metab. 20, 158-171 (2014).

44. Yang, M., Zhang, Y. \& Ren, J. Autophagic Regulation of Lipid Homeostasis in Cardiometabolic Syndrome. Front. Cardiovascular Med. 5, 38 (2018).

45. Liu, T. Y. et al. FNDC5 alleviates hepatosteatosis by restoring AMPK/mTORmediated autophagy, fatty acid oxidation, and lipogenesis in mice. Diabetes 65, 3262-3275 (2016)

46. Piacentini, M. et al. Non-alcoholic fatty liver disease severity is modulated by transglutaminase type 2. Cell Death Dis. 9, 257 (2018).

47. Zheng, M. et al. Cardiac-specific ablation of Cypher leads to a severe form of dilated cardiomyopathy with premature death. Hum. Mol. Genet. 18, 701-713 (2009).

48. Cheng, H., Lederer, W. J. \& Cannell, M. B. Calcium sparks: elementary events underlying excitation-contraction coupling in heart muscle. Science $\mathbf{2 6 2}$, 740-744 (1993).

49. Shang, W. et al. Cyclophilin D regulates mitochondrial flashes and metabolism in cardiac myocytes. J. Mol. Cell. Cardiol. 91, 63-71 (2016).

50. Fan, F. et al. Deletion of heat shock protein 60 in adult mouse cardiomyocytes perturbs mitochondrial protein homeostasis and causes heart failure. Cell Death Differ. 27, 587-600 (2019).

51. Shen, T. et al. Mitofusin-2 is a major determinant of oxidative stress-mediated heart muscle cell apoptosis. J. Biol. Chem. 282, 23354-23361 (2007).

52. Wisniewski, J. R., Zougman, A., Nagaraj, N. \& Mann, M. Universal sample preparation method for proteome analysis. Nat. Methods 6, 359-362 (2009).

53. Huang da, W., Sherman, B. T. \& Lempicki, R. A. Bioinformatics enrichment tools: paths toward the comprehensive functional analysis of large gene lists. Nucleic Acids Res. 37, 1-13 (2009). 